\title{
Limit distribution of the size of the giant component in a web random graph
}

\author{
Yuri Pavlov $\|^{1 /}$ \\ ${ }^{1}$ Karelian Research Center of the Russian Academy of Sciences, 24/8 - 28, Kirova str., 185000, Petrozavodsk, Russia
}

Consider random graph with $N+1$ vertices as follows. The degrees of vertices $1,2, \ldots, N$ are the independent identically distributed random variables $\xi_{1}, \xi_{2}, \ldots, \xi_{N}$ with distribution

$$
\mathbf{P}\left\{\xi_{1} \geq k\right\}=k^{-\tau}, \quad k=1,2, \ldots, \quad \tau \in(1,2),
$$

and the vertex $N+1$ has degree 0 , if the sum $\zeta_{N}=\xi_{1}+\ldots+\xi_{N}$ is even, else degree is 1 . From (1) we get that

$$
p_{k}=\mathbf{P}\left\{\xi_{1}=k\right\}=k^{-\tau}-(k+1)^{-\tau}, \quad k=1,2, \ldots
$$

Let $G\left(k_{1}, \ldots, k_{N}\right)$ be a set of graphs with $\xi_{1}=k_{1}, \ldots, \xi_{N}=k_{N}$. If $g$ is a realization of random graph then

$$
\mathbf{P}\left\{g \in G\left(k_{1}, \ldots, k_{N}\right)\right\}=p_{k_{1}} \cdot \ldots \cdot p_{k_{N}} .
$$

The probability distribution on the set of graph is defined such that for a vector $\left(k_{1}, \ldots, k_{N}\right)$ all graphs, lying in $G\left(k_{1}, \ldots, k_{N}\right)$, are equiprobable. Studies of the past few years show that such graphs are good random graph models for Internet and other networks topology description (see, for example, H. Reittu and I. Norros (2004)).

To build the graph, we have $N$ numbered vertices and incident to vertex $i \xi_{i}$ stubs, $i=1, \ldots, N$. All stubs need to be connected to another stub to construct the graph. The stubs are numbered in an arbitrary order from 1 to $\zeta_{N}$.

Let $\eta_{(N)}$ be the maximum degree of the vertices. Hence

$$
\eta_{(N)}=\max _{1 \leq i \leq N} \xi_{i}
$$

Theorem 1 Let $N \rightarrow \infty$. Then

$$
\mathbf{P}\left\{\tau \ln \eta_{(N)}-\ln N<x\right\}=e^{-e^{-x}}+o(1) .
$$

We denote by $\varphi(z)$ the generating function

$$
\varphi(z)=\sum_{k=1}^{\infty} p_{z} z^{k}
$$

It is easy to see that $\mathbf{E} \xi_{1}=\zeta(\tau)$, where $\zeta(\cdot)$ is the Riemann's zeta-function. Let $\xi^{*}$ be the random variable with distribution:

$$
\mathbf{P}\left\{\xi^{*}=k\right\}=(k+1) p_{k+1} / \zeta(\tau), \quad k=0,1,2, \ldots
$$

We consider the Galton-Watson branching process $\mu(t)$ with one initial particle and offspring distribution (2). Let $q$ be the extinction probability of the $\mu(t)$. We denote by $i^{*}$ the vertex with degree $\eta_{(N)}$. H. Reittu and I. Norros (2004) proved that $i^{*}$ belongs to giant connected component and the size $\nu$ of this component divided by $N$ tends to $1-\varphi(q)$ as $N \rightarrow \infty$.

Let $A=A^{(N)}$ be an event that a vertex belongs to giant component. It is known that $\mathbf{P}\{A\} \rightarrow 1-\varphi(q)$. The limit distribution of the variable $\nu$ is described by the following theorem.

Theorem 2 Let $N \rightarrow \infty$. Then

$$
\mathbf{P}\left\{\frac{N \mathbf{P}\{A\}-\nu}{\sqrt{N \varphi(q)(1-\varphi(q))}}<x\right\} \rightarrow \frac{1}{\sqrt{2 \pi}} \int_{-\infty}^{x} e^{-y^{2} / 2} d y .
$$

\section{References}

H. Reittu, I. Norros. On the power-law random graph model of massive data networks. Performance Evaluation, v.55, 2004, 3-23.

${ }^{\dagger}$ The work is supported by RFBR, grant 05-01-00007a. 
